\title{
Toxoplasmose: análise do conhecimento em mulheres na cidade de Guaraí/TO
}

A toxoplasmose é causada pelo protozoário Toxoplasma gondii, e alcança altos índices de infecção no Brasil. O parasita possui formas infectantes que permitem que o homem e outras espécies de animais se contaminem de diversas formas como, a congênita ou transplacentária, por manuseio de fezes de gatos, ingestão de carne crua ou malpassada, entre outras. No entanto, a infecção pode ser combatida através de medidas preventivas, e estas estão intimamente ligadas a higiene e hábitos da população. Dessa forma o estudo teve como objetivo, analisar o conhecimento sobre a toxoplasmose e consequentemente abordar as medidas preventivas em mulheres, em idade reprodutiva ( 10 a 49 anos), que residem em Guaraí - TO, porem somente mulheres acima dos 18 anos participaram da pesquisa. A escolha da análise ser feita somente com mulheres em idade reprodutiva, foi dado pelo fato de que estas possuem alto risco de infecção por toxoplasmose, considerando que a forma mais grave de contração é a congênita. As mesmas foram submetidas a um questionário composto por 12 questões relacionadas a toxoplasmose. Constatou-se que as pesquisadas em sua maioria desconhecem a doença e que o fator escolaridade influência de maneira positiva, visto que mulheres com maior nível de escolaridade apresentaram melhores resultados quando questionadas sobre a temática, além de demostrar a falta de orientação médica, pelo fato de muitas desconhecerem os testes laboratoriais de diagnóstico.

Palavras-chave: Toxoplasmose; Conhecimento; Transmissão; Prevenção.

\section{Toxoplasmosis: analysis of knowledge in women in the city of Guaraí/TO}

\begin{abstract}
Toxoplasmosis is caused by the protozoan Toxoplasma gondii, and achieves high rates of infection in Brazil. The parasite has infecting forms that allow man and other animal species to become contaminated in various ways, such as congenital or transplacental, by handling cat feces, ingestion of raw or malpasted meat among others. However, the infection can be combated through preventive measures, and these are closely linked to the hygiene and habits of the population. Thus, the study aimed to analyze the knowledge about toxoplasmosis and consequently address preventive measures in women, of reproductive age (10 to 49 years), who live in Guarai - TO, but only women over 18 years participated in the research. The choice of the analysis to be made only with women of reproductive age was made by the fact that they have a high risk of toxoplasmosis infection, considering that the most severe form of contraction is congenital. They were submitted to a questionnaire composed of 12 questions related to toxoplasmosis. It was found that the majority of the studies are unaware of the disease and that the schooling factor influences positively, since women with a higher level of education presented better results when questioned about the theme, besides showing the lack of medical guidance, due to the fact that many do not know the laboratory diagnostic tests.
\end{abstract}

Keywords: Toxoplasmosis; Knowledge; Streaming; Prevention.

Topic: Ensino Superior, Pesquisa e Extensão

Reviewed anonymously in the process of blind peer
Received: 02/02/2021

Approved: 27/02/2021
Mateus Silva Santos (iD

Instituto Educacional Santa Catarina, Brasil http://lattes.cnpq.br/4059367759608845 http://orcid.org/0000-0002-0811-8869 biomateus07@outlook.com

Isalara Cespedes Correa (iD)

Instituto Educacional Santa Catarina, Brasil

http://lattes.cnpq.br/5318143728058517

http://orcid.org/0000-0002-6013-9448

isalaracespedescorrea@gmail.com

Jean da Silva Oliveira (iD)

Instituto Educacional Santa Catarina, Brasil

http://lattes.cnpq.br/3965762157612466

http://orcid.org/0000-0002-8927-2517

isalaracespedescorrea@gmail.com

\section{Referencing this:}

SANTOS, M. S.; CORREA, I. C.; OLIVEIRA, J. S.. Toxoplasmose: análise do conhecimento em mulheres na cidade de Guaraí/TO. Revista Ibero Americana de Ciências Ambientais, v.12, n.2, p.687-698, 2021. DOI: http://doi.org/10.6008/CBPC2179-6858.2021.002.0057 


\section{INTRODUÇÃO}

A estreita relação que há entre o ser humano e o meio ambiente e consequentemente com as variedades de espécies de animais, mais habitualmente com cães e gatos, faz com que o mesmo se torne mais acessível a adquirir doenças infecto-parasitarias, como a toxoplasmose (RODRIGUES, 2015). Esta enfermidade é de grande importância na saúde pública, sendo uma das zoonoses parasitarias mais disseminadas no mundo (TENTER, 2009).

A toxoplasmose é uma infecção causada por um protozoário intracelular obrigatório, denominado Toxoplasma gondii, (FLEGR et al., 2014). Este possui diversos hospedeiros intermediários, porem somente os felídeos são hospedeiros definitivos, pois são nestes animais que o protozoário consegue completar seu ciclo de vida (MILLAR et al., 2012; HILL et al., 2016).

O T. gondii, foi descoberto pela primeira vez em 1908 pelos parasitologistas franceses, Charles Nicolle e Louis Herbert Manceaux em tecidos de um roedor africano silvestre, o Ctenodactylus gundi. Por se tratar de um roedor que era utilizado em estudos de leishmaniose, estes acreditaram que este microrganismo era do gênero Leishmania, mas ao ser observado, constatou-se que se tratava de um novo organismo e o nomeou de T. gondii, baseado na sua morfologia (toxo: arco, plasma: vida) e no seu hospedeiro. No Brasil, em São Paulo, Alfonso Splendore na mesma época encontrou o protozoário em um coelho, porem o identificou erroneamente como Leishmania (NICOLLE et al., 1909; DUBEY, 2016; MOURA et al., 2016).

A primeira descrição de toxoplasmose congênita em humanos foi constatada em uma criança que foi a óbito aos 11 meses com hidrocefalia e cegueira, cujo em sua necropsia foi localizado parasitas parecidos ao T. gondii, pelo oftalmologista Jankü (JANKÜ, 1923). No Brasil, o primeiro caso de toxoplasmose congênita foi descrito no Rio de Janeiro, por Torres (1927), onde foram encontrados vestígios do parasita em cortes histológicos do cérebro, miocárdio e musculo esquelético de um recém-nascido que veio a óbito no 29 dia de vida (TORRES, 1927).

De acordo com Moura et al. (2016), somente em 1948, criou-se por Sabin e Feldman o teste do corante de Sabin-Feldman, que permitiu que diversos investigadores estudassem os aspectos clínicos e epidemiológicos da toxoplasmose, mostrando assim que está infecção possui alta prevalência no mundo e é caracterizada por ser assintomática nos pacientes.

Em 1965, Hutchison reconheceu o gato como participante no ciclo evolutivo do parasita, revelando que esses animais são capazes de elimina-los pelas fezes (HUTCHISON, 1967). No entanto, em 1976, foi comprovado que o gato não era o único responsável por transmitir o protozoário (KAWAZOE, 2005).

O T. gondii apresenta ampla distribuição geográfica, pois pode ser encontrado em todo planeta (ELMORE et al., 2010), além de mostrar que é um dos principais patógenos, pelo fato de sua alta dominância, principalmente em todos os animais homeotérmicos e possui grande potencial patogênico nos seres humanos (DUBEY, 2016; SARAF et al., 2017).

Estima-se que cerca de 1/3 da população mundial já foi infectada por T. gondii. e as taxas de infecção podem variar de região para região devido a diversos fatores tais como clima, hábitos higiênicos, hábitos de 
preparação e ingestão de alimento, à sua baixa virulência e a sua rápida multiplicação (BLADER et al., 2015).

Segundo Dubey (2016), os felídeos, principalmente os gatos são os hospedeiros definitivos deste protozoário, pois nestes são capazes de se reproduzirem de forma sexuada e assexuada diferente dos hospedeiros intermediários, onde o parasita só se reproduz assexuadamente.

As três principais vias de transmissão da toxoplasmose para seres humanos são por ingestão de carne crua ou malcozida contaminada, contato com as fezes de gatos, solo ou alimentos contaminados e infecção do feto via transplacentária/congênita (MITSUKA-BREGANÓ et al., 2010).

Segundo Branco et al. (2012), a infecção por toxoplasmose é geralmente assintomática, em 80 a 90\% dos indivíduos imunocompetentes, já nos imunocomprometidos e, especialmente os recém-nascidos, são observadas formas graves da doença. Geralmente pessoas com imunidade alta não apresentam sintomas, no entanto indivíduos que possuem condições especiais de imunidade como os portadores da Síndrome de Imunodeficiência Humana (AIDS), a infecção por toxoplasmose na fase aguda, pode comprometer o sistema nervoso, pulmonar, cardíaco, intestinal, muscular e ocular (RODRIGUES, 2015).

O problema maior ocorre em mulheres gestantes, pois estas quando expostas pela primeira vez ao parasito, não possuem anticorpos maternos, o que pode leva-la ao aborto espontâneo, nascimento prematuro, morte neonatal, ou o feto pode nascer com sequelas severas (AMENDOEIRA et al., 2010).

A gestação é o período onde o feto está em formação e desenvolvimento, como a toxoplasmose pode acarretar graves consequências, é necessário diagnosticar precocemente essas gestantes, para que seja efetuado o devido tratamento (MONTAÑO et al., 2010). Durante o primeiro trimestre, a infecção pode levar o feto a morte, no segundo trimestre, pode causar a chamada Tétrade de Sabin, onde a criança pode apresentar calcificações cerebrais, retardo mental, hidrocefalia com macro ou microcefalia e perturbações neurológicas e após o parto, a criança pode vir a apresentar apenas evidências da doença como: febre, cegueira e manchas pelo corpo ou ainda pode nascer e se desenvolver normalmente e vir a apresentar sintomas da infecção somete quando mais velhas (SOUZA et al., 2010).

Existem diversos testes que auxiliam na identificação da toxoplasmose, porém os testes sorológicos mais comuns em humanos são a imunofluorescência indireta e ELISA, onde são observados os anticorpos das classes IgM e IgG anti-T. gondii. O diagnóstico precoce durante o período gestacional é essencial para que se dê início ao tratamento em caso de confirmação, e é feito pelo perfil da doença em situação aguda, que analisa anticorpos IgM e IgG. (MONTAÑO et al., 2010).

Apesar do tratamento controlar a proliferação rapidamente, não há nenhuma droga capaz de extinguir por completo os cistos teciduais de animais e humanos, além disso, em casos de imunocomprometimento a toxoplasmose pode ser reativada (MONTAÑO et al., 2010). O Ministério da Saúde (2010), mostrou altos índices dessa doença no Brasil e ao mesmo tempo a necessidade de haver conhecimento sobre a prevenção e as formas de transmissão da toxoplasmose pela população, especialmente as mulheres.

Devido à ausência de estudos sobre o tema em questão, na cidade de Guaraí -TO, o presente artigo tem como objetivo relatar o conhecimento das mulheres sobre a toxoplasmose e consequentemente 
abordar o meio de transmissão e prevenção do mesmo, já que não existem vacinas ou medicações preventivas para o controle do parasita.

\section{MATERIAIS E MÉTODOS}

Esse estudo é de cunho quantitativo-qualitativo que se baseou em coleta de dados e por demonstração estatística dos resultados obtidos.

\section{Características do local de estudo}

O município de Guaraí, está localizado na região norte do Estado do Tocantins nas coordenadas 8o $50^{\prime} 4^{\prime \prime}$ de latitude sul e $48^{\circ} 30^{\prime} 36^{\prime \prime}$ de longitude oeste com altitude de 254 metros, possui uma área de $2268,16 \mathrm{~km}^{2}$ (Figura 1) e que segundo o último censo tem uma população de aproximadamente 23.200 habitantes. Guaraí foi escolhida para realização do estudo, tendo em vista que está possui clima favorável para o parasita, o que beneficia a permanência de oocistos no solo, sendo assim a população fica tendente a contaminações, e ainda, somando-se o fato da vigilância sanitária do município não notificar o caso da infecção.

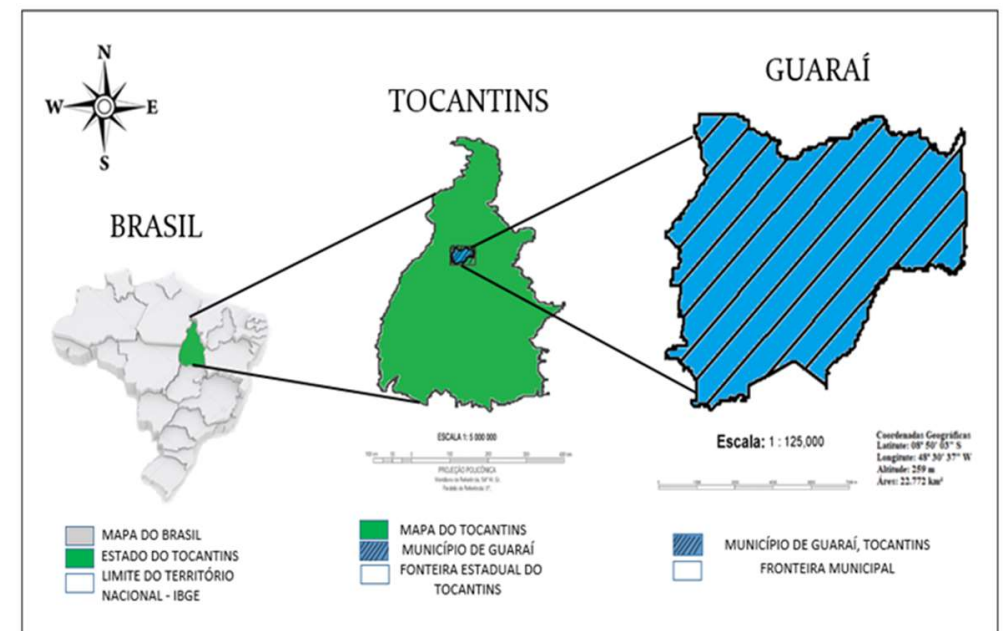

Figura 1. Mapa do estado do Tocantins, dando destaque ao município de Guaraí (TO). Fonte: Silva et al. (2018).

\section{Critérios de elegibilidade}

A pesquisa ocorreu de maneira aleatória pelo município de Guaraí, nas residências dos pesquisados, abrangendo diferentes classes sociais e diferentes níveis de escolaridade. A análise foi realizada com cento e cinco (105) mulheres, que residem no município, que se encontravam na faixa etária classificada pelo Ministério de Saúde como idade reprodutiva (10-49 anos), porem participaram somente mulheres acima de 18 anos, por estas poderem assinar o Termo de Consentimento Livre e Esclarecido (TCLE). A escolha de mulheres na faixa etária reprodutiva, foi feita por estas possuírem alto risco de infecção da toxoplasmose, considerando-se que uma das formas mais graves de contração da doença é a transplacentária, no qual a mãe transmite para o feto durante a gestação. 


\section{Metodologia de aplicação}

Foi aplicado um questionário, onde Gil (2002) e Prodanov et al. (2013), compreendem que questionário é um conjunto de questões onde estas serão respondidas pelo pesquisado. O questionário utilizado continha doze (12) perguntas fechadas de múltipla escolha, que segundo Freitas et al. (2019), facilita a interpretação das perguntas e auxilia na análise de dados. Todas as perguntas eram de autoria própria, porem baseadas em outros artigos já publicados acerca da mesma temática.

Foram estipuladas doze perguntas semiestruturadas contidas no questionário aplicado para as mulheres de Guaraí (TO) acerca da toxoplasmose, para análise do conhecimento das mesmas (Quadro 1).

Quadro 1: Questões utilizadas no questionário aplicado as mulheres acerca da toxoplasmose.

\begin{tabular}{|l|}
\hline Idade? \\
\hline Qual o seu grau de escolaridade? \\
\hline Você já ouviu falar em toxoplasmose? \\
\hline Sabe o que toxoplasmose? \\
\hline Você sabe como é realizado o diagnóstico da toxoplasmose? \\
\hline Você já realizou o exame de sague para detectar a toxoplasmose (IgG)? \\
\hline Você acha que a toxoplasmose pode ser transmitida de mãe para filho? \\
\hline Possui gato? \\
\hline Você sabia que animais domésticos, principalmente os gatos, podem ser portadores do parasita responsável pela toxoplasmose? \\
\hline $\begin{array}{l}\text { Se sim, qual maneira você achar que pode ser a mais eficaz para evitar que o seu animal de estimação seja portador do parasita } \\
\text { responsável pela toxoplasmose? }\end{array}$ \\
\hline Você tem filhos? \\
\hline Se sim, durante o seu período de acompanhamento (pré-natal), o seu médico te orientou sobre a toxoplasmose? \\
\hline
\end{tabular}

\section{RESULTADOS E DISCUSSÃO}

Foram avaliadas o conhecimento sobre toxoplasmose em 105 mulheres, residentes do município de Guaraí - TO. A tabela 1, demonstra que 47 (44,76\%), das mulheres pesquisadas possuíam de 35 a 49 anos, seguindo de $32(30,47 \%)$ com idade de 18 a 25 anos. Dentre essas, 44 (41,90\%) das pesquisadas possuíam o ensino médio completo.

Tabela 1: Dados referentes a idade e escolaridade das mulheres pesquisadas em Guaraí - TO, 2019.

\begin{tabular}{lll}
\hline Variável & Total de participantes & $\%$ \\
\hline Faixa etária (anos) & 32 & $30,47 \%$ \\
$18-25$ & 26 & $24,76 \%$ \\
$26-34$ & 47 & $44,76 \%$ \\
$35-49$ & & $15,23 \%$ \\
Escolaridade & 16 & $1,90 \%$ \\
Fundamental incompleto & 02 & $12,38 \%$ \\
Fundamental completo & 13 & $41,90 \%$ \\
Ensino Médio Incompleto & 44 & $19,04 \%$ \\
Ensino Médio completo & 20 & $9,52 \%$ \\
Superior incompleto & 10 & \\
Superior completo & &
\end{tabular}

\section{Conhecimento sobre o termo toxoplasmose}

Segundo Rodrigues (2015), acredita-se que conhecer é o primeiro passo para a construção de uma opinião crítica e reflexiva sobre as formas e medidas preventivas da toxoplasmose. Borguezan et al. (2014), afirma que o conhecimento sobre a infecção e os fatores de risco que possam estar operando em diversas regiões nos mais variados grupos populacionais, é de suma importância para que haja a implantação de 
medidas preventivas, como programas de atenção básica de risco. A tabela 2 apresenta o percentual de conhecimento das mulheres em relação a toxoplasmose.

Tabela 2: Conhecimento das mulheres sobre a toxoplasmose em Guaraí - TO, 2019.

\begin{tabular}{lll}
\hline Perguntas & Sim (\%) & Não (\%) \\
\hline Você já ouviu falar de toxoplasmose? & $73(69,52 \%)$ & $32(30,47 \%)$ \\
Sabe o que é toxoplasmose? & $59(56,19 \%)$ & $46(43,80 \%)$ \\
\hline
\end{tabular}

Os dados apresentados na tabela, demostram um baixo nível de conhecimento relacionado a toxoplasmose, gerando uma certa preocupação, pois não conhecer sobre o assunto implica na qualidade de vida como um todo. Resultados semelhantes foram encontrados na pesquisa de Ribeiro (2010), com estudantes de escolas do ensino fundamental em Belo Horizonte, este estudo demonstrou que os educandos apresentavam pouco conhecimento sobre zoonoses em geral e em específico, a toxoplasmose.

No estudo de Barbosa et al. (2009) em que avaliaram o conhecimento de mulheres grávidas, 68,9\% das gestantes atendidas, não conheciam a toxoplasmose sendo que cerca de $49,5 \%$ possuíam sorologia positiva para anti-T. godii. Em seu estudo ainda afirmaram que a falta de conhecimento é um dos principais fatores de risco para contrair a infecção.

Um estudo realizado na Polônia mostrou que em quatro anos, após o início de atividades de educação em saúde, o conhecimento da população sobre os fatores de risco do T. godii, praticamente dobrou (PAWLOWSKI et al., 2001). Já em outro estudo realizado na Bélgica, demonstrou que após ser realizada a educação em saúde houve uma redução de 63,0\% na taxa de soroconversão materna (FOULON et al., 1994).

No Brasil, a prática de educação é bastante mencionada, mas pouco valorizada (BRANCO et al., 2012). Entretanto, no ano de 2009 avanços foram observados o município de Londrina - PR, ao ser desenvolvido e implantado um programa de vigilância em saúde para toxoplasmose gestacional e congênita, e a educação em saúde é um de seus pilares, esse programa trouxe melhoras no atendimento de gestantes e crianças com suspeita ou confirmação de toxoplasmose (MITSUKA-BREGANÓ et al., 2010).

No presente estudo, foi relacionado o conhecimento das mulheres sobre toxoplasmose com o nível de escolaridade (Tabela 3), observando resultados positivos quanto a interferência da escolaridade no processo de identificar e conhecer doenças.

Tabela 3: A escolaridade relacionada ao conhecimento das mulheres sobre toxoplasmose em Guaraí - TO, 2019.

\begin{tabular}{lll}
\hline \multicolumn{1}{c}{ Escolaridade } & Não sabe & Sabe \\
\hline Fundamental incompleto & 12 & 04 \\
Fundamental completo & 01 & 01 \\
Ensino Médio incompleto & 07 & 06 \\
Ensino Médio completo & 20 & 24 \\
Superior incompleto & 04 & 16 \\
Superior completo & 01 & 09 \\
\hline
\end{tabular}

Na tabela 3, pode ser observado que mulheres com um maior nível de escolaridade (ensino médio completo - superior completo), conseguem desenvolver resultados positivos ao serem questionadas sobre o tema. Resultados semelhantes foram encontrados na pesquisa de Rodrigues (2015), onde 14,59\% das mulheres com maior nível de escolaridade de ensino médio completo à pós-graduação, obtiveram melhores resultados em comparação a mulheres que nunca frequentaram escola ou que possuem ensino médio 
incompleto.

Santos (2019) em sua pesquisa afirma que existe de maneira geral um baixo nível de conhecimento sobre a toxoplasmose, principalmente nos grupos de pessoas mais jovens ou de baixa escolaridade. No estudo realizado por Tome et al. (2010) relacionado ao conhecimento da toxoplasmose, evidenciou que $84,68 \%$ dos analisados não sabiam conceituar a doença, e que $84,08 \%$ desconheciam as formas de contágio.

Em seu trabalho Moura et al. (2018), aponta que o baixo nível escolar e consequentemente a falta de informação sobre a toxoplasmose torna as gestantes propensas a contaminação e logo a transmissão transplacentária. Quites (2009) e Barbosa et al. (2009), relacionaram que quanto menor o nível de escolaridade, maior é o índice de infecção, dessa forma por não obter conhecimento sobre a toxoplasmose favorece uma maior exposição aos fatores de risco por não os conhecer.

\section{Conhecimento relacionado ao diagnóstico e transmissão congênita da toxoplasmose}

Tendo em vista que a prevenção contra a infecção congênita depende de um diagnóstico precoce materno (RODRIGUES, 2015), no questionário foi colocado questões que investigava se as mulheres possuíam conhecimento referente ao teste para detecção da toxoplasmose e se as mesmas já realizaram o exame de identificação de anticorpos IgG (Tabela 4).

Segundo Sartori et al. (2011) a triagem sorológica para anticorpos anti-T. gondii deve fazer parte da rotina dos serviços de saúde pré-natal, pois a ausência de anticorpos IgG permite identificar gestantes suscetíveis. Estas deverão receber orientações sobre os fatores de risco e medidas preventivas durante a gestação, além de realizar acompanhamento do status sorológico.

Através do questionário, que tinha como público alvo as mulheres, foi avaliado se a toxoplasmose pode ser transmitida de forma congênita (transplacentária), visto que a transmissão da infecção pode ocorrer de mãe para filho. Silveira (2001), em seu estudo afirma que nos EUA a cada mil nascimentos 2 casos de toxoplasmose são confirmados, já no Brasil em média seis mil crianças nascem por ano com esta infecção, pelo fato da maioria das mulheres não receberem acompanhamento sorológico adequado.

Tabela 4: Conhecimento das mulheres em relação ao exame e orientação médica em Guaraí - TO, 2019.

\begin{tabular}{|c|c|c|c|}
\hline Perguntas & $\operatorname{Sim}(\%)$ & Não (\%) & Não sabe (\%) \\
\hline Você sabe como é realizado o diagnóstico da toxoplasmose? & $47(44,76 \%)$ & $58(55,23 \%)$ & \\
\hline Você já realizou exame de sangue para detectar toxoplasmose (IgG)? & $27(25,71 \%)$ & $50(47,61 \%)$ & $28(26,66 \%)$ \\
\hline Você tem filhos? & $81(77,14 \%)$ & $24(22,85 \%)$ & \\
\hline $\begin{array}{l}\text { Se sim, durante o seu período de acompanhamento (pré-natal), o seu } \\
\text { médico te orientou sobre a toxoplasmose? }\end{array}$ & $55(52,38 \%)$ & $27(25,71 \%)$ & $23(21,90 \%)$ \\
\hline Você acha que a toxoplasmose pode ser transmitida de mãe para filho? & $54(51,42 \%)$ & $34(32,38 \%)$ & $17(16,19 \%)$ \\
\hline
\end{tabular}

Questionadas se sabiam como era realizado o diagnóstico para toxoplasmose, $58(55,23 \%)$ responderam não terem conhecimento e $47(44,76 \%)$ responderam saber. Quando questionadas se já teriam realizado o teste de identificação de anticorpos IgG anti $-T$. gondii, $50(47,61 \%)$ afirmaram não terem realizado o teste, $28(26,66 \%)$ disseram que não sabiam se em algum momento da vida tinha realizado o exame e somente $27(25,71 \%)$ informaram que já realizaram o exame.

Dados semelhantes foram encontrados na pesquisa de Rodrigues (2015), em relação ao teste de IgG, 
no mesmo $87,5 \%$ das mulheres responderam não conhecer e apenas $12,5 \%$ afirmaram conhecer e quanto ao terem feito o teste de $73,69 \%$ responderam não saber, $15,62 \%$ não tinham feito e apenas $10,67 \%$ responderam já terem feito. Para Rodrigues (2015), isso demonstra a grande falta de conhecimento da população sobre o exame sorológico, pois este é o meio mais simples e seguro para detectar a infecção, e esta deve ser realizada para constatar anticorpos IgM e IgG, na primeira consulta de pré-natal e será repetindo nos meses seguintes, sendo um exame obrigatório por toda gestante.

O número mínimo de consultas para que o pré-natal seja considerado apropriado, no Brasil é de seis meses, e a sorologia além de acontecer na primeira consulta deve continuar a cada trimestre (BRASIL, 2005). No entanto, o teste sorológico de rotina é realizado somente uma vez na primeira consulta de pré-natal na maioria dos casos no Brasil (AMENDOEIRA et al., 2010).

Com base nos dados pode-se observar que 55 (52,38\%) das entrevistadas disseram ter recebido orientação médica, outras 27 (25,71\%) afirmam que não recebeu, já 23 (21,90\%) delas não souberam responder. Em geral esses dados demonstram notoriamente uma falta de instrução médica aos pacientes. De acordo com Silva et al. (2012), a orientação sobre a toxoplasmose realizada como método de prevenção da transmissão do $T$. gondii durante a gravidez pode reduzir os riscos da obtenção da infecção durante a gestação.

Das 105 mulheres analisadas, 81 responderam terem filhos e destas apenas 27 afirmaram terem sido orientadas sobre toxoplasmose durante o pré-natal. Amendoeira et al. (2010), identificaram que existe um grande número de gestantes que não receberam informações durante o pré-natal ou só receberam acompanhamento no final do terceiro trimestre de gravidez, em casos como esses, se o teste sorológico identificar anticorpos maternos específicos, será mais complexo descobrir se a mulher adquiriu a infecção durante a gestação.

Em relação à forma de transmissão transplacentária, 54 (51,42\%) responderam ser transmitida de mãe para filho, 34 (32,38\%) disseram que não, e outros 17 (16,19\%) não souberam responder. É notório uma porcentagem grande de mulheres que não conhecem essa forma de transmissão. Vale ressaltar que também pode ser adquirida através do manuseio de fezes de felinos, manuseio de solo (areia) e consumo de carne crua ou malpassada, (SARTORI et al., 2011).

Rodrigues (2015), supõe que essa falta de conhecimento pode ser explicada pela falta de orientação médica/profissional. Fato comprovado por Branco et al. (2012) que em sua pesquisa sobre o conhecimento e atitudes dos profissionais da saúde na prevenção da toxoplasmose mostrou que os profissionais não informavam as gestantes sobre o teste sorológico, apenas solicitavam. No mesmo estudo, ao serem questionados sobre qual conduta a ser tomada caso uma gestante apresentasse IgM e IgG anti-T gondii reagentes, $77,48 \%$ dos técnicos de enfermagem,77,14\% dos enfermeiros e $69,70 \%$ dos médicos não responderam ou deram uma resposta incorreta.

\section{Conhecimento e medidas preventivas relacionadas a animais de estimação}

Ao serem questionadas sobre a criação de animais domésticos, 48 (45,71\%) das mulheres relatou 
possuir gato de estimação, e 57 (54,28\%) relataram que não possuem. É importante ressaltar que a grande maioria das pesquisadas sabiam que o gato é o portador principal do parasita, porém conheciam a toxoplasmose pelo nome popular, "doença do gato". Das mulheres participantes 65 (61,90\%) afirmaram que tinha conhecimento sobre o tema e $40(38,09 \%)$ responderam que não sabiam. Estes dados podem ser observados logo abaixo, na tabela 5 .

Tabela 5: Análise de conhecimento relacionado ao gato como principal portador da toxoplasmose em Guaraí - TO, 2019.

\begin{tabular}{|c|c|c|c|}
\hline Perguntas & Sim (\%) & Não (\%) & Não sabe (\%) \\
\hline Possui gato? & $48(45,71 \%)$ & $57(54,28 \%)$ & \\
\hline $\begin{array}{l}\text { Você sabia que animais domésticos, principalmente os gatos, podem ser } \\
\text { portadores do parasita responsável pela toxoplasmose? }\end{array}$ & 65 (61,90\%) & $40(38,09 \%)$ & \\
\hline
\end{tabular}

Esses dados não afirmam que a falta de animais de estimação é uma medida preventiva, porém é importante verificar quem possuem esses animais, para identificar se os mesmos praticam cuidados apropriados, quanto à higiene e alimentação do animal como medidas preventivas.

Quites (2009), por meio de seus estudos afirmou que o fato de ter animais dentro ou fora da residência não causa infecção pelo T. gondii. Para Carvalho (2011), a presença do gato em casa pode ser considerada um fator de risco, se não haver hábitos de higiene adequados por parte dos donos, já que esses animais são os únicos capazes de eliminar oocistos nas fezes e estão presentes no cotidiano de muitas famílias.

Ao serem questionados sobre como evitar que o animal de estimação adquira toxoplasmose (tabela 6), notou-se que a maioria 44 (42\%) marcou a alternativa referente a vacinação e 17 (16\%) marcaram a alternativa sobre o livre acesso do animal a rua, o que auxilia o animal não adquirir a infecção, pois em caso de gatos que possuem livre acesso, estes são mais propensos a adquirir a doença, já que possivelmente terá contato com outros ambientes, logo terão acesso a ratos contaminados, podendo assim adquirir o parasita.

Tabela 6: Medidas preventivas relacionado aos cuidados com gatos de estimação com mulheres de Guaraí - TO, 2019.

\begin{tabular}{lll}
\hline \multicolumn{2}{l}{ Mediadas } & Medidas realizadas pelas participantes (\%) \\
\hline a) & Evitar oferecer carne crua ao animal. & $18(17,14 \%)$ \\
b) & Evitar que o animal tenha acesso livre a rua. & $17(16,19 \%)$ \\
c) & Não há como prevenir. & $7,61 \%)$ \\
d) & Vacinação do animal & $44(41,90 \%)$ \\
e) & Limpeza do ambiente em que o animal vive. & $18(17,14 \%)$ \\
\hline
\end{tabular}

Em comparação as demais alternativas, as opções referentes a alimentação e limpeza do local onde o animal vive, foram as menos marcadas, mostrando um resultado negativo, visto que a ingestão de carne crua e a falta de limpeza do ambiente são fatores, que influenciam como um meio de transmissão ao animal. Montanõ et al. (2010), menciona que os gatos que geralmente ingerem carne crua ou malpassada, na maioria das vezes se infectam por cistos de toxoplasma.

Para Dabritz et al. (2010), os proprietários de gatos, devem mantê-los dentro de suas residências e coletar diariamente suas fezes como forma de controle, evitando que se tornem infectantes. Rodrigues (2015), reafirma que o ambiente em que o animal vive deve permanecer limpo, e as fezes devem ser retiradas e colocadas em sacos plásticos para que não haja contaminação. 
Grande parte das pesquisadas marcaram a alternativa que corresponde a vacinação do animal, demostrando um ponto positivo, a favor da vacinação como meio preventivo, porém mostra a falta de informação perante ao assunto. O estudo realizado por Santos et al. (2018), demonstrou que 20\% dos pesquisados também colocaram a vacinação como medida de prevenção, no entanto não existem vacinas eficazes contra toxoplasmose.

Segundo Rodrigues (2015), atualmente não existe vacinas para seres humanos, já para os animais a ideia ainda está sendo estudada na tentativa de reduzir os cistos teciduais e danos fatais a animais de produção, tornando-se de grande interesse econômico. Freire et al. (2003), diz que as vacinas estão sendo criadas, com proposito de prevenir que os felinos eliminem oocistos através das fezes, que consequentemente diminuirá a contaminação do ambiente, e a infecção de animais de produção, assim como a infecção transplacentária.

\section{CONCLUSÕES}

Baseado nos resultados encontrados na pesquisa, foi observado que as mulheres analisadas em sua maioria não possuem informação relacionados a toxoplasmose, apesar da importância dessa doença. Comparando o conhecimento das pesquisadas com nível de escolaridade foi notado que quanto maior a escolaridade maior a tendência de conhecer a toxoplasmose e suas formas de transmissão.

O fato que contribui para o aumento da infecção e consequentemente gasto público com a enfermidade é justamente a falta de informação sobre toxoplasmose e suas formas de transmissão. Notouse que, a maior parte das mulheres, não tem conhecimento do teste de IgG, pelo fato de não terem realizado o exame ou não saberem responder se em algum momento já realizaram, indicado assim também a falta de orientação médica durante o pré-natal, pois a maioria (81) das mulheres possuem filhos e destas somente 27 afirmaram ter sido orientadas durante a gestação.

Torna-se imprescindível o papel dos profissionais da saúde na população, em específico as mulheres, visando o esclarecimento das medidas preventivas, com isso as futuras gestantes poderiam se prevenir amenizando os casos da infecção e consequentemente os riscos causados aos fetos.

\section{REFERÊNCIAS}

AMENDOEIRA, M. R. R.; CAMILLO-COURA, L. F. C.. Uma breve revisão sobre toxoplasmose na gestação. Scien. Med., v.20, n.1, p.113-119, 2010

BARBOSA, I. R.; CARVALHO, X. H. C. M.; ANDRADE NETO, V. F.. Toxoplasmosis screening and risk factors amongst pregnant females in Natal, northeastern Brazil. Trans. R. Soc. Trop. Med. Hyg., v.103, n.4, p.377-382, 2009. DOI: https://doi.org/10.1016/j.trstmh.2008.11.025

BORGUEZAN, C.; SANCHES, F. G.; OLIVEIRA, J. T. M.; NORBERG, P. R. B. M.; URIARTE, M. A. A.; NORBERG, A. N.. Seroprevalencia De Anticuerpos Anti-Toxoplasma Gondii En Indígenas De La Etnia Terena, Mato Grosso Do Sul, Brasil. Revista Cubana de Medicina Tropical, Rio de Janeiro, v.66, n.1, p.48-57, 2014.
BLADER, I. J.; COLEMAN, B. I.; CHEN, C. T.; GUBBELS, M. Lytic cycle of Toxoplasma gondii: 15 years later. Annual Review of Microbiology, v; 69, p.463-485, 2015. DOI: http://doi.org/101146/annurev-micro-091014-104100

BRANCO, B. H. M.; ARAÚJO, S. M. D.; FALAVGNAGUILHERME, A. L.. Prevenção primária da toxoplasmose: conhecimento e atitudes de profissionais de saúde e gestantes do serviço público de Maringá, estado do Paraná. Sci. Med., v.22, n.4, p.185-190, 2012.

BRASIL. Ministério da Saúde. Secretaria de Atenção à Saúde. Pré-natal e puerpério: atenção qualificada e humanizada. Brasília: MS, 2005. 
CARVALHO, L. H.. Avaliação do conhecimento das gestantes sobre toxoplasmose em uma unidade de atendimento obstétrico na cidade de Formiga - MG. Monografia (Bacharelado em Medicina Veterinária) - Centro Universitário de Formiga, Formiga, 2011

DABRITZ, H. A.; CONRAD, P. A.. Cats and Toxoplasma: implications for public health. Zoonoses and Public Health, v.57, p.34-52, 2010. DOI: https://doi.org/10.1111/j.18632378.2009.01273.x

DUBEY, J. P.. Toxoplasmosis of animals and humans. CRC press, 2016

ELMORE, S. A.; JONES, J. L.; CONRAD, P. A.; PATTON, S.; LINDSAY, D. S.; DUBEY, J. P.. Toxoplasma gondii: epidemiology, feline clinical aspects, and prevention. Trends in Parasitology, v.26, n.4, p.190-196, 2010. DOI: https://doi.org/10.1016/j.pt.2010.01.009

FOULON, W.; NAESSENS, A.; DERDE, P.. Evoluation of the possibities for preventing congenital toxoplasmosis. American Journal of Perinatology, n.11, p.57-62, 1994.

FLEGR, J.; PRANDOTA, J.; SOVIČKOVÁ, M.; ISRAILI, Z. H.. Toxoplasmosis-a global threat. Correlation of latent toxoplasmosis with specifc disease burden in a set of 88 countries. PloS One, v.9, n.3, p.e90203, 2014. DOI: https://doi.org/10.1371/journal.pone.0090203

FREIRE, R. L.; NAVARRO, I. T.; BRACARENSE, A. P. F. R. L.; GENNARI, S. M.. Vaccination of pigs with Toxoplasma gondii antigens incorporated in immunostimulating complexes (iscoms). Arquivo Brasileiro de Medicina Veterinária e Zootecnia, v.55, n.4, p.388-396, 2003. DOI: https://doi.org/10.1590/S0102-09352003000400002

FREITAS, K. T. S.; BARROS, J. B.; CARVALHO, A. V.. Controle e prevenção de pulgas em cães e gatos: análise das ações dos tutores. Agriculturae, v.1, n.1, p.13-20, 2019. DOI: https://doi.org/10.6008/CBPC2674-645X.2019.001.0002

GIL, A. C.. Como elaborar projetos de pesquisa. São Paulo, v.5, n.61, p.16-17, 2002.

HILL, D. E.; DUBEY, J. P.. Toxoplasma gondii as a Parasite in Food: Analysis and Control. Microbiology spectrum, v.4, n.4, 2016.

HUTCHISON, W. M.. The nematode transmission of Toxoplasma gondii. Trans. Roy Soc. Trop. Med. Hyg, v.61, n.1, p.80-89, 1967. DOI: https://doi.org/10.1016/0035$\underline{\text { 9203(67)90056-9 }}$

JANKU, J.. Pathogenesis and pathologic anatomy of the coloboma of the macula lutea in an eye of normal dimensions and in a microphithalmic eye with parasites in the reting. Cas Lek Cesk, v.61, p.1021-52, 1923.

KAWAZOE, U.. Toxoplasma gondii In: NEVES, D. P.. Parasitologia Humana. São Paulo: Atheneu, 2005. p.163172.

MILLAR, P. R.; ALVES, F. M.; TEIXEIRA, V. Q.; VICENTE, R. T.; MENEZES, E. M.; SOBREIRO, L. G.; AMENDOEIRA, M. R.. Ocorrência da infecção por toxoplasma gondii e fatore associados à sua transmissão em aves de corte e postura produzidas em diferentes de criação. Pesquisa Veterinária Brasileira, v.32, n.3, p.79-88, 2012. DOI: http://dx.doi.org/10.1590/S0100-736X2012000300009

MITSUKA-BREGANÓ, R.; LOPES-MORI, F. M. R.; NAVARRO, I. T.. Toxoplasmose adquirida na gestação e congênita: vigilância em saúde, diagnostico, tratamento e condutas. Eduel, 2010. DOI: https://doi.org/10.7476/9788572166768

MONTAÑO, P. Y.; CRUZ, M. D. A.; ULLMANN, L. S.; LANGONI, H.; BIONDO, A. W.. Contato com gatos: um fator de risco para a toxoplasmose congênita?. Clínica Veterinária, v.86, p.78-84, 2010

MOURA, F. L. D.; GOULART, P. R. M.; MOURA, A. P. P. D.; SOUZA, T. S. D.; FONSECA, A. B. M.; AMENDOEIRA, M. R. R.. Fatores associados ao conhecimento sobre a toxoplasmose entre gestantes atendidas na rede pública de saúde do município de Niterói, Rio de Janeiro, 2013-2015.

Epidemiologia e Serviços de Saúde, v.25, p.655-661, 2016. DOI: http://doi.org/10.5123/S1679-49742016000300022

MOURA, D. S.; OLIVEIRA, R. C. M.; MATOS, R. T. J.. Toxoplasmose gestacional: perfil epidemiológico e conhecimentos das gestantes atendidas na unidade básica de saúde de um município alagoano. Arquivos Médicos dos Hospitais e da Faculdade de Ciências Médicas da Santa Casa de São Paulo, v.63, n.2, p.69-76, 2018. DOI: https://doi.org/10.26432/1809-3019.2018.63.2.69

NICOLLE, C.; MANCEAUX, L.. Sur un protozoaire nouveau du gondi. C R Acad. Sci., v.27, p.148-369, 1909.

PAWLOWSKI, Z. S.; GROMADECKA-SUTKIEWICZ, M.; SKOMMER, J.; PAUL, M.; ROKOSSOWSKI, H.; SUCHOCKA, E.; SCHANTZ, P. M.. Impact of health education on knowledge and prevention behavior for congenital toxoplasmosis: the experience in Poznan, Poland. Health Education Research, n.16, p.493-502, 2001.

PRODANOV, C. C.; FREITAS, E. C.. Metodologia do trabalho científico: métodos e técnicas da pesquisa e do trabalho acadêmico. 2 ed. Feevale, 2013.

QUITES, H. F. O.. Fatores associados à infecção com toxoplasma gondii em comunidade rural do Vale do Jequitinhonha, Minas Gerais. Dissertação (Mestrado em Saúde e Enfermagem) - Universidade Federal de Minas Gerais, Belo Horizonte, 2009.

RIBEIRO, L. M. L.. Analise do conhecimento, sobre leishmaniose visceral e outras zoonoses, de docentes dos três primeiros anos do ensino fundamental em escolas da região noroeste de Belo Horizonte, Minas Gerais, 2008. Dissertação (Mestrado em Ciência Animal) - Universidade Federal de Minas Gerais, Belo Horizonte, 2010.

RODRIGUES, D. N. J.. Avaliação do Conhecimento da População sobre Formas de Tansmissão e Medidas Preventivas da Toxoplasmose em Mossoró - RN. Dissertação (Metrado em Ambiente, Tecnologia e Sociedade) - Universidade Federal do Semiárido, Mossoró, 2015.

SANTOS, N. A. D.. Avaliação do nível de conhecimento sobre a toxoplasmose na comunidade escolar do Instituto Federal de Mato Grosso - Campus Confresa. Monografia 
(Bacharelado em Farmácia) - Universidade Federal Mato Grosso, Barra do Garças, 2019.

SANTOS, E. W.; SOUZA, S. L.; PEREIRA, C. A. D.. Gato $x$ gestante. Avaliação do conhecimento da população sobre a toxoplasmose. PUBVET, v.12, p.136, 2018. DOI:

https://doi.org/10.31533/pubvet.v12n12a238.1-5

SARAF, P.; SHWAB, E. K.; DUBEY, J. P.; SU, C.. On the determination of Toxoplasma gondii virulence in mice. Experimental Parasitology, v.174, p.25-30, 2017. DOI: https://doi.org/10.1016/i.exppara.2017.01.009

SARTORI, A. L.; MINAMISAVA, R.; AVELINO, M. M.; MARTINS, C. A.. Triagem pré-natal para toxoplasmose e fatores associados à soropositividade de gestantes em Goiânia, Goiás. Revista Brasileira de Ginecologia e Obstetrícia, v.33, n.2, 2011. DOI: https://doi.org/10.1590/S010072032011000200007

SILVA, B. B.; ROCHA, L. G.; SILVEIRA, L. P. O.; CARVALHO, A. V.; GUIMARÃES, A. P. M.. Análise de qualidade da água do Ribeirão Tranqueira. Revista Ibero-Americana de Ciências Ambientais, v.9, n.8, p.11-25, 2018. DOI: http://doi.org/10.6008/CBPC2179- 6858.2018.008.0002

SILVA, L. R.; OKAZAKI, E. L. F. J.. Enfermagem e a prevenção da Toxoplasmose durante a gestação. Revista de Enfermagem da Universidade de Santo Amaro, v.13 n.1, p.43-47, 2012

SILVEIRA, C.. Toxoplasmosis - Literature review (1997 to 2000). Arq. Bras. de Oftalmo, v.64, p.70-84, 2001.

SOUZA, C. D. O.; TASHIMA, N. T.; SILVA, M. A. D.; TUMITAN, A. R. P.. Estudo transversal de toxoplasmose em alunas de um curso superior da região de Presidente Prudente, Estado de São Paulo. Revista da Sociedade Brasileira de Medicina Tropical, v.43, n.1, p.59-61, 2010. DOI: https://doi.org/10.1590/S0037-86822010000100013

TENTER, A. M.. Toxoplasma gondii in animals used for human consumption. Memórias do Instituto Oswaldo Cruz, v.104, p.364-369, 2009. DOI: https://doi.org/10.1590/S007402762009000200033

TOME, R. O.; LANGONI, H.; PERUCA, L. C. B.; BABBONI, S. D. Avaliação do Conhecimento Sobre Algumas Zoonoses com Proprietários de Cães da Área Urbana do Município de Botucatu-SP. Journal of Health Sciences, v.12, n.3, 2010. DOI: https://doi.org/10.17921/2447-8938.2010v12n3p\%25p

TORRES, C. M.. Sur une nouvelle maladie de l'homme, charactérisée par la présence d'une parasite intracellulaire, très proche de Toxoplasma et de l'Encephalitozoon, dans le tissu musculaire cardique, les muscles du squelette, le tissu cellulair sourcutane et le tissu ne. Comptes Rendus Biologies, v.97, p.1778-1781, 1927.

A CBPC - Companhia Brasileira de Produção Científica (CNPJ: 11.221.422/0001-03) detém os direitos materiais desta publicação. Os direitos referem-se à publicação do trabalho em qualquer parte do mundo, incluindo os direitos às renovações, expansões e disseminações da contribuição, bem como outros direitos subsidiários. Todos os trabalhos publicados eletronicamente poderão posteriormente ser publicados em coletâneas impressas sob coordenação da Sustenere Publishing, da Companhia Brasileira de Produção Científica e seus parceiros autorizados. Os (as) autores (as) preservam os direitos autorais, mas não têm permissão para a publicação da contribuição em outro meio, impresso ou digital, em português ou em tradução. 